\title{
Effect of flow fluctuations and nonflow on elliptic flow methods
}

\author{
Jean-Yves Ollitrault, ${ }^{1}$ Arthur M. Poskanzer, ${ }^{2}$ and Sergei A. Voloshin ${ }^{3}$ \\ ${ }^{1}$ CNRS, URA2306, Institut de physique théorique de Saclay, F-91191 Gif-sur-Yvette, France \\ ${ }^{2}$ Lawrence Berkeley National Laboratory, Berkeley, California, 94720 \\ ${ }^{3}$ Wayne State University, Detroit, Michigan, 48201
}

(Dated: April 16, 2009)

\begin{abstract}
We discuss how the different estimates of elliptic flow are influenced by flow fluctuations and nonflow effects. It is explained why the event-plane method yields estimates between the twoparticle correlation methods and the multiparticle correlation methods. It is argued that nonflow effects and fluctuations cannot be disentangled without other assumptions. However, we provide equations where, with reasonable assumptions about fluctuations and nonflow, all measured values of elliptic flow converge to a unique mean $v_{2, \mathrm{PP}}$ elliptic flow in the participant plane and, with a Gaussian assumption on eccentricity fluctuations, can be converted to the mean $v_{2, \mathrm{RP}}$ in the reaction plane. Thus, the $20 \%$ spread in observed elliptic flow measurements from different analysis methods is no longer mysterious.
\end{abstract}

PACS numbers: 25.75.Ld, 24.10.Nz

\section{INTRODUCTION}

Elliptic flow has proved to be very valuable for the understanding of relativistic nuclear collisions [1]. However, different analysis methods give results which spread over a range of $20 \%$ [2]. A higher accuracy is now needed because when comparing to relativistic viscous hydrodynamic calculations, an uncertainty of $30 \%$ in the elliptic flow parameter $v_{2}$ leads to an uncertainty of $100 \%$ in the ratio of shear viscosity to entropy [3]. The experimental measurements need to converge to allow extraction of such important characteristics of the matter produced in relativistic nuclear collisions. The problem of nonflow correlations contributing to $v_{2}$ has been known for a long time [4]. More recently it has been recognized that fluctuations affect the measured $v_{2}$ values $[5,6]$. It is now also recognized that some measurements are relative to the participant plane and some to the reaction plane [7]. The reaction plane is spanned by the vector of the impact parameter and the beam direction. The participants are those constituents which partake in the primary interaction. The minor axis of the participant zone and the beam direction define the participant plane. The event plane contains the flow vector $Q$ constructed from the transverse momenta of the detected particles.

The purpose of this paper is to propose a method using reasonable assumptions to obtain a well-defined measure of elliptic flow to compare with theoretical calculations. Section II describes the flow analysis methods which we will be discussing. The analytic equations are derived in Secs. III and IV, and summarized in Sec. V. Tests of the equations by numerical integrations and simulations are in Sec VI. Then the analytic equations are applied to published STAR data in Sec. VII. Sec. VIII is a summary.

For simplicity we will write $v\{\}$ instead of $v_{n}\{\}$ and $\cos (\ldots)$ instead of $\cos [n(\ldots)]$, where $n$ is the harmonic number of the anisotropic flow. The final equations are independent of $n$.

\section{FLOW METHODS}

The two-particle cumulant method $v\{2\}$ correlates each particle with with every other particle, and is defined as [8]

$$
v\{2\} \equiv \sqrt{\left\langle\cos \left(\phi_{1}-\phi_{2}\right)\right\rangle},
$$

where \langle\rangle indicates an average over all particles in all events. The four-particle cumulant method $v\{4\}$ is defined as [9]

$v\{4\} \equiv\left(2\left\langle\cos \left(\phi_{1}-\phi_{2}\right)\right\rangle^{2}-\left\langle\cos \left(\phi_{1}+\phi_{2}-\phi_{3}-\phi_{4}\right)\right\rangle\right)^{1 / 4}$.

The Lee-Yang Zeros method [10] $v\{L Y Z\}$ is also a multiparticle correlation.

The event-plane method $v_{2}\{\mathrm{EP}\}$ correlates each particle with the event plane of the other particles. The event-plane azimuth $\Psi_{R}$ is defined as the azimuthal angle of the flow vector:

$$
\begin{aligned}
\mathbf{q} \cos \Psi_{R} & =\frac{\mathbf{Q}}{\sqrt{N}} \cos \Psi_{R}=\frac{1}{\sqrt{N}} \sum_{j=1}^{N} \cos \phi_{j} \\
\mathbf{q} \sin \Psi_{R} & =\frac{\mathbf{Q}}{\sqrt{N}} \sin \Psi_{R}=\frac{1}{\sqrt{N}} \sum_{j=1}^{N} \sin \phi_{j}
\end{aligned}
$$

where $|q| \geq 0$ and the sum runs over particles defining the event plane. Since $Q$, the magnitude of the standard flow vector, is proportional to $\sqrt{N}$ in the absence of correlations, it is convenient to use $\mathbf{q} \equiv \mathbf{Q} / \sqrt{N}$. The event-plane estimate of anisotropic flow is defined as

$$
v\{\mathrm{EP}\} \equiv \frac{\left\langle\cos \left(\phi-\Psi_{R}\right)\right\rangle}{R},
$$

where the particle of interest is always subtracted from q before calculating $\Psi_{R}$ in order to avoid autocorrelations. $R$ is the event plane resolution correction which is 
determined from the correlation between the event plane vectors of two independent "subevents" $A$ and $B$. In the original method $[11,12]$, subevent $A$ is defined by choosing randomly $N / 2$ particles out of the $N$ particles of the event plane and subevent $B$ is made of the remaining $N / 2$ particles. Other methods of choosing the subevents are now also used, such as according to pseudorapidity or charge, or combinations of these. For sake of generality, we denote by $N_{s}$ the number of particles in a subevent. The azimuths $\Psi_{A}$ and $\Psi_{B}$ are defined by equations similar to Eq. (3), where $N$ is replaced with $N_{s}$.

In the special case where the event plane is made of only one subevent $N=N_{s}$, the resolution correction is the subevent resolution:

$$
R=\sqrt{\left\langle\cos \left(\Psi_{A}-\Psi_{B}\right)\right\rangle} .
$$

The corresponding estimate of anisotropic flow will be denoted by $v\{$ subEP $\}$, or more particularly, $v\{$ etaSub $\}$ or $v\{$ ranSub\}, depending on how the events were divided.

In the more general case when the event plane is larger than one subevent, one first estimates the resolution parameter $\chi_{s}$ of the subevents by solving numerically the equation

$$
\mathcal{R}\left(\chi_{s}\right)=\sqrt{\left\langle\cos \left(\Psi_{A}-\Psi_{B}\right)\right\rangle},
$$

where the function $\mathcal{R}$ is defined by $[11,13,14]$

$$
\mathcal{R}(\chi)=\frac{\sqrt{\pi}}{2} e^{-\chi^{2} / 2} \chi\left(I_{0}\left(\frac{\chi^{2}}{2}\right)+I_{1}\left(\frac{\chi^{2}}{2}\right)\right),
$$

where $I_{0}$ and $I_{1}$ are modified Bessel functions. Generally, the resolution parameter is related to the flow through

$$
\chi_{s}=v \sqrt{N_{s}}
$$

One then estimates the resolution parameter $\chi$ of the full event as $\chi \equiv \chi_{s} \sqrt{N / N_{s}}$. The resolution correction for the full event $R$ is defined by

$$
R \equiv \mathcal{R}(\chi)=\mathcal{R}\left(\chi_{s} \sqrt{N / N_{s}}\right)
$$

If the event plane coincides with one subevent $\chi=\chi_{s}$, and Eqs. (6) and (9) reduce to (5).

For a review of anisotropic flow see Ref. [1].

\section{FLUCTUATIONS}

Elliptic flow is driven by the initial eccentricity of the overlap almond [15]. This eccentricity fluctuates from one event to the other. There are several sources of fluctuations: fluctuations of impact parameter within the sample of events [16], and more importantly, fluctuations of the positions of participant nucleons $[5,6,17]$. It is fluctuations which make $\langle v\rangle$ in the participant plane larger than in the reaction plane. The magnitude of flow fluctuations is characterized by $\sigma_{v}$, defined by

$$
\sigma_{v}^{2} \equiv\left\langle v^{2}\right\rangle-\langle v\rangle^{2}
$$

where $v$ is the flow in the participant plane $v_{\mathrm{PP}}$ in the case of fluctuations in the participant plane. Flow methods involve various functions of $v$, which are also affected by fluctuations. The average value of $f(v)$ is obtained by expanding around $\langle v\rangle$ to leading order in $\sigma_{v}^{2}$ :

$$
\langle f(v)\rangle=f(\langle v\rangle)+\frac{\sigma_{v}^{2}}{2} f^{\prime \prime}(\langle v\rangle) .
$$

This result will be useful below.

We now derive the effect of fluctuations on the various flow estimates, to order $\sigma_{v}^{2}$. Using the definitions of $v\{2\}$ and $v\{4\}$ from Eqs. (1) and (2),

$$
v\{2\}^{2}=\left\langle v^{2}\right\rangle=\langle v\rangle^{2}+\sigma_{v}^{2}
$$

and

$$
\begin{aligned}
v\{4\}^{2} & =\left(2\left\langle v^{2}\right\rangle^{2}-\left\langle v^{4}\right\rangle\right)^{1 / 2} \\
& \approx\langle v\rangle^{2}-\sigma_{v}^{2}
\end{aligned}
$$

Fluctuations increase $v\{2\}$ and decrease $v\{4\}$ compared to $v_{\mathrm{PP}}$. In the case of Gaussian eccentricity fluctuations, $v\{4\}$ measures the correlation to the true reaction plane $v_{\text {RP }}[7,18]$. However, it has been shown that eccentricity fluctuations are not quite Gaussian, especially for peripheral collisions [7, 19].

The contribution of fluctuations to the various $v\{\}$ results can be parameterized by $\alpha$ [19]:

$$
v\{\}=\left\langle v^{\alpha}\right\rangle^{1 / \alpha} .
$$

Eq. (11) with $f(v)=v^{\alpha}$ gives

$$
\left\langle v^{\alpha}\right\rangle=\langle v\rangle^{\alpha}\left(1+\frac{\sigma_{v}^{2}}{\langle v\rangle^{2}} \frac{\alpha(\alpha-1)}{2}\right)
$$

Raising to the power $2 / \alpha$ and expanding to leading order in $\sigma_{v}^{2}$,

$$
v\{\}^{2}=\langle v\rangle^{2}+(\alpha-1) \sigma_{v}^{2} .
$$

Note that $v\{4\}$ from Eq. (13) corresponds to the limiting case $\alpha=0$ and $v\{2\}$ from Eq. (12) corresponds to the case $\alpha=2$. The event plane methods have intermediate $\alpha$.

We now derive the value of $\alpha$ for $v\{$ subEP $\}$, defined by Eqs. (4) and (5). The subevent resolution depends on the flow $v$, which fluctuates:

$$
v\{\operatorname{subEP}\}^{2}=\frac{\langle v \mathcal{R}(v)\rangle^{2}}{\left\langle\mathcal{R}^{2}(v)\right\rangle} .
$$

The averages in the numerator and in the denominator can be evaluated using Eq. (11). Expanding to leading order in $\sigma_{v}^{2}$, one obtains

$$
v\{\mathrm{subEP}\}^{2}=\langle v\rangle^{2}+\frac{\langle v\rangle \mathcal{R}^{\prime}}{\mathcal{R}}\left[2-\frac{\langle v\rangle \mathcal{R}^{\prime}}{\mathcal{R}}\right] \sigma_{v}^{2},
$$


where $\mathcal{R}^{\prime}$ is the derivative of $\mathcal{R}$ with respect to $v$. Comparing to (16), one obtains the following expression of $\alpha$, which is independent of $\sigma_{v}$ :

$$
\alpha=1+\frac{\langle v\rangle \mathcal{R}^{\prime}}{\mathcal{R}}\left[2-\frac{\langle v\rangle \mathcal{R}^{\prime}}{\mathcal{R}}\right] .
$$

Inserting the expression (9) for $\mathcal{R}(\chi)$ and using the fact that $\chi$ is proportional to $v$, one obtains after some algebra

$$
\alpha=2-\frac{4 i_{1}^{2}}{\left(i_{0}+i_{1}\right)^{2}},
$$

where $i_{0,1}$ is a shorthand notation for $I_{0,1}\left(\chi_{s}^{2} / 2\right)$.

As an example of the application of Eq. 20 we re-plot Ref. [19] Fig. 5 as Fig. 1 here. Alpha is defined by Eq. (14) and the resolution is the subevent plane resolution. Simulations including event-by-event fluctuations were done and and analyzed with the subevent method, and using Eq. (14) alpha was extracted. Our Eq. (20) has been added to the figure without any adjustable parameters. The extraordinary fit means that fluctuations quantitatively explain the figure.

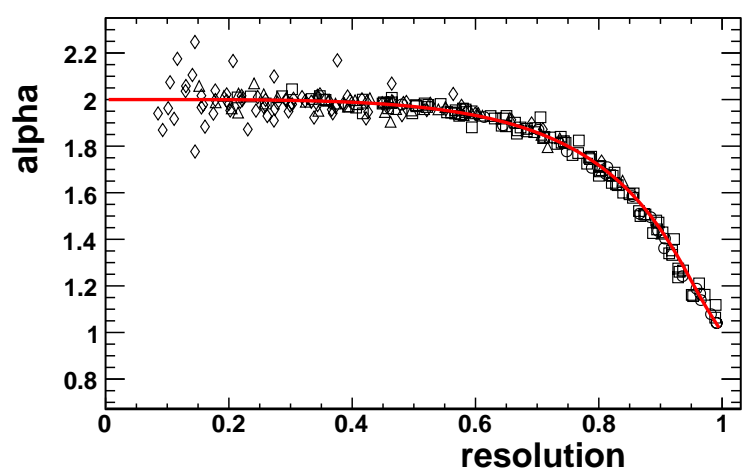

FIG. 1: (Color online) Alpha, defined in Eq. (14), vs the subevent plane resolution. The simulations of $v_{2}\{$ subEP $\}$ are from Ref. [19]. The solid line is Eq. (20).

Inserting Eq. 20 into Eq. (16), one gets

$$
v\{\operatorname{subEP}\}^{2}=\langle v\rangle^{2}+\left(1-\frac{4 i_{1}^{2}}{\left(i_{0}+i_{1}\right)^{2}}\right) \sigma_{v}^{2} .
$$

The case where the event plane consists of two subevents is studied in appendix A. Eq. (21) is replaced by the more general expression

$$
\begin{aligned}
& v\{\mathrm{EP}\}^{2}=\langle v\rangle^{2} \\
& \quad+\left(1-\frac{I_{0}-I_{1}}{I_{0}+I_{1}}\left(2 \chi^{2}-2 \chi_{s}^{2}+\frac{4 i_{1}^{2}}{i_{0}^{2}-i_{1}^{2}}\right)\right) \sigma_{v}^{2}
\end{aligned}
$$

where again $i_{0,1}$ is a shorthand notation for $I_{0,1}\left(\chi_{s}^{2} / 2\right)$ for subevents, while $I_{0,1}$ is a shorthand notation for
$I_{0,1}\left(\chi^{2} / 2\right)$ for the whole event. The corresponding expression for $\alpha$ is obtained again by comparing to Eq. (16):

$$
\alpha=2-\frac{I_{0}-I_{1}}{I_{0}+I_{1}}\left(2 \chi^{2}-2 \chi_{s}^{2}+\frac{4 i_{1}^{2}}{i_{0}^{2}-i_{1}^{2}}\right) .
$$

When the event plane consists of one subevent only, $\chi=$ $\chi_{s}, I_{0,1}=i_{0,1}$, and Eqs. (22) and (23) reduce to (21) and (20), respectively.

\section{NONFLOW EFFECTS}

In this section we discuss nonflow effects while neglecting fluctuations. $v$ can therefore be identified with $\langle v\rangle$. The two-particle azimuthal correlation gets contributions from flow and from other, "nonflow" effects:

$$
\left\langle\cos \left(\phi_{1}-\phi_{2}\right)\right\rangle \equiv\langle v\rangle^{2}+\delta
$$

where $\delta$ is the nonflow part. One expects that $\delta$ varies with centrality like $1 / N$, where $N$ is some measure of the multiplicity $[4,11]$.

Using Eqs. (1) and (24), one obtains, to leading order in $\delta$ :

$$
v\{2\}^{2}=\langle v\rangle^{2}+\delta
$$

On the other hand, $v\{4\}$ is insensitive to nonflow effects, thus

$$
v\{4\}=\langle v\rangle \text {. }
$$

We now derive the expression of $v\{\mathrm{EP}\}$ to leading order in $\delta$. In the same way as fluctuations, nonflow effects contribute to both the numerator and denominator of Eq. (4). These contributions are evaluated in detail in Appendix B. The nonflow correlation between the particle and the event plane (numerator) is derived by shifting the flow vector by an amount proportional to $\delta$, and to the unit vector of the particle. The nonflow correlation between subevents is taken into account in the probability distribution of $\mathbf{q}_{\mathbf{A}}, \mathbf{q}_{\mathbf{B}}$ by a correlation term, whose form is dictated by the central limit theorem. One must also take into account the fact that nonflow correlations modify the width of fluctuations of the flow vector around the reaction plane, which are responsible for the resolution correction in Eq. (4). One obtains

$$
\begin{aligned}
& v\{\mathrm{EP}\}^{2}=\langle v\rangle^{2} \\
& \quad+\left(1-\frac{I_{0}-I_{1}}{I_{0}+I_{1}}\left(\chi^{2}-\chi_{s}^{2}+\frac{2 i_{1}^{2}}{\left(i_{0}^{2}-i_{1}^{2}\right)}\right)\right) \delta .
\end{aligned}
$$

If the event plane consists of only one subevent, $I_{0,1}=$ $i_{0,1}, \chi=\chi_{s}$, and this simplifies to

$$
v\{\mathrm{subEP}\}^{2}=\langle v\rangle^{2}+\left(1-\frac{2 i_{i}^{2}}{\left(i_{0}+i_{1}\right)^{2}}\right) \delta .
$$

If the resolution is low, $i_{1} \ll i_{0}$ and $v\{$ subEP $\}$ coincides with $v\{2\}$, Eq. (25). If the resolution is large, $i_{1} \simeq i_{0}$ and $v\{$ subEP $\}$ lies half-way between $v\{2\}$ and $v\{4\}$. 


\section{SUMMARY OF EQUATIONS}

We assume that to leading order in $\sigma_{v}^{2}$ and $\delta$, the contributions of nonflow and fluctuations are additive. Eqs. (12) and (25) yield:

$$
v\{2\}^{2}=\langle v\rangle^{2}+\delta+\sigma_{v}^{2} .
$$

Similarly, Eqs. (13) and (26) yield

$$
v\{4\}^{2}=\langle v\rangle^{2}-\sigma_{v}^{2}
$$

Although this equation was derived for $v_{2}\{4\}$ it should apply to all multiparticle values. As for the event-plane method, Eqs. (22) and (27) give

$$
v\{\mathrm{EP}\}^{2}=\langle v\rangle^{2}+\left(1-\frac{\left(I_{0}-I_{1}\right)}{\left(I_{0}+I_{1}\right)}\left(\chi^{2}-\chi_{s}^{2}+\frac{2 i_{1}^{2}}{\left(i_{0}^{2}-i_{1}^{2}\right)}\right)\right) \delta+\left(1-\frac{2\left(I_{0}-I_{1}\right)}{I_{0}+I_{1}}\left(\chi^{2}-\chi_{s}^{2}+\frac{2 i_{1}^{2}}{i_{0}^{2}-i_{1}^{2}}\right)\right) \sigma_{v}^{2} .
$$

Finally, Eqs. (21) and (28) yield

$$
v\{\operatorname{subEP}\}^{2}=\langle v\rangle^{2}+\left(1-\frac{2 i_{1}^{2}}{\left(i_{0}+i_{1}\right)^{2}}\right) \delta+\left(1-\frac{4 i_{1}^{2}}{\left(i_{0}+i_{1}\right)^{2}}\right) \sigma_{v}^{2} .
$$

This can be derived from Eq. 31 by setting $\chi=\chi_{s}$.

The difference between estimates always scales like $\delta+2 \sigma_{v}^{2}$ :

$$
\begin{aligned}
v\{2\}^{2}-v\{4\}^{2} & =\delta+2 \sigma_{v}^{2} \\
v\{2\}^{2}-v\{\mathrm{EP}\}^{2} & =\frac{\left(I_{0}-I_{1}\right)}{\left(I_{0}+I_{1}\right)}\left(\chi^{2}-\chi_{s}^{2}+\frac{2 i_{1}^{2}}{\left(i_{0}^{2}-i_{1}^{2}\right)}\right)\left(\delta+2 \sigma_{v}^{2}\right) \\
v\{2\}^{2}-v\{\mathrm{subEP}\}^{2} & =\frac{2 i_{1}^{2}}{\left(i_{0}+i_{1}\right)^{2}}\left(\delta+2 \sigma_{v}^{2}\right) .
\end{aligned}
$$

Thus we have defined $\sigma_{\text {tot }}^{2} \equiv \delta+2 \sigma_{v}^{2}$. This shows explicitly that fluctuations and nonflow effects cannot be disentangled with only these measurements.

\section{NUMERIC INTEGRATIONS AND SIMULATIONS}

To avoid the leading order expansions in Sec. III, one can test the accuracy of the analytic equations by performing numeric integrations or analyzing simulations to solve for the various $v\{\}$ quantities from $\langle v\rangle$ and $\sigma_{v}$ in the participant plane. If one assumes a Gaussian distribution there is a tail to negative values of $v$ for large fluctuations. Since participant eccentricity never goes negative, one can avoid this problem by using a Bessel-Gaussian distribution. Also, assuming a two-dimensional Gaussian in the reaction plane makes the distribution along the participant plane axis have the form of a Bessel-Gaussian [7]:

$$
\frac{d n}{d v}=\frac{v}{\sigma_{0}^{2}} I_{0}\left(\frac{v v_{0}}{\sigma_{0}^{2}}\right) \exp \left(-\frac{v^{2}+v_{0}^{2}}{2 \sigma_{0}^{2}}\right),
$$

where $v_{0}$ and $\sigma_{0}$ are parameters of the distribution which are adjusted so that the first and second moments equal $\langle v\rangle$ and $\sigma_{v}$. The equations for these moments are in Ref. [7]. The relative magnitude of fluctuations is maximum for $v_{0}=0$, corresponding to zero impact parameter central collisions: $\sigma_{v} /\langle v\rangle=\sqrt{(4 / \pi)-1}=52.2 \%$ [20].

\section{A. Numeric}

For the subevent plane and full event plane flow values we evaluate from Eq. (17)

$$
\begin{gathered}
v\{\operatorname{subEP}\}=\frac{\langle v \mathcal{R}(v \sqrt{N / 2})\rangle}{\sqrt{\left\langle[\mathcal{R}(v \sqrt{N / 2})]^{2}\right\rangle}} \\
v\{\mathrm{EP}\}=\frac{\langle v \mathcal{R}(v \sqrt{N})\rangle}{\mathcal{R}\left[\mathcal{C}\left(\sqrt{\left\langle[\mathcal{R}(v \sqrt{N / 2})]^{2}\right\rangle}\right) \sqrt{2}\right]},
\end{gathered}
$$

where $\mathcal{R}$ and $\mathcal{C}$ are functions which calculate the event plane resolution and resolution parameter $\chi$, respectively. $\mathcal{R}$ is given by Eq. (7) and $\chi$ is solved from that equation by iteration. The averages are taken by integrating over the normalized Bessel-Gaussians from 0 to $v_{0}+4 \sigma_{0}$. For central collisions, $v_{0}=0$ and integrations can be done analytically, as shown in Appendix A 2. For this zero impact parameter case, Figure 2 displays the ratio of the exact values of $v\{\mathrm{subEP}\}$ and $v\{\mathrm{EP}\}$ to the approximate expressions derived in Sec. III. This figure shows that for realistic values of the resolution, the formulas in Sec. III for maximum fluctuations are valid within $1 \%$.

For the nonflow dependence, adding $\delta / 2$ to $\sigma_{v}^{2}$ would only take into account the broadening of the distribution, and not the direct nonflow correlations. Thus the effect of nonflow was tested only by simulations. 


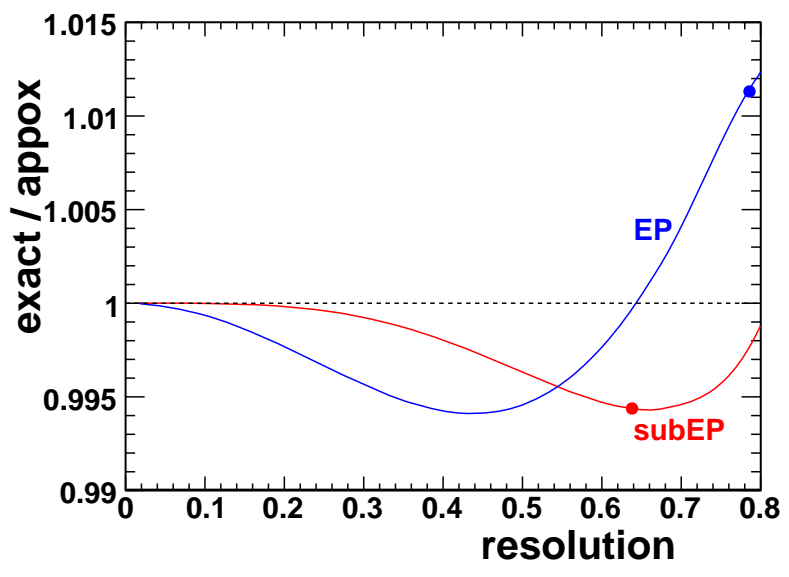

FIG. 2: (Color online) Ratio of the exact values of $v\{$ subEP $\}$ and $v\{\mathrm{EP}\}$, defined by Eqs. (35) and (36), to the approximate expressions (21) and (22), for central collisions, versus the reaction plane resolution. The $\mathrm{x}$-axis is the full event resolution for $v\{\mathrm{EP}\}$ and the subevent resolution for $v\{\mathrm{subEP}\}$. The points correspond to the points in Fig. 3. However, for central collisions the resolution is normally lower than this.

\section{B. Simulations}

The simulation results for fluctuations were obtained by generating 8 million events of fixed multiplicity $=400$, and elliptic flow values uniformly distributed in the range of 0 to 0.2 . The angle of each track was selected randomly according to the azimuthal distribution defined by the elliptic flow of that event. After all tracks were generated they were divided into two equal subevents and the corresponding flow vectors generated. The event plane resolution, the observed flow, and the final flow values, were calculated by applying a weight to each event according to a Bessel-Gaussian distribution with parameters which produced $\langle v\rangle=0.06$ and a corresponding $\sigma_{v}$ for plotting vs $\sigma_{v} /\langle v\rangle$.

The non-flow effects were simulated by generating similar events of fixed multiplicity $=200$ without flow and different numbers of pairs of particles with exactly the same azimuthal angle. If $f$ is the fraction of all particles generated as pairs with the same azimuth, $\delta=f / N$, where $N$ is the full multiplicity.

\section{Tests of the equations}

For fixed $\langle v\rangle$ in the participant plane, the corrections for the analytic method from Sec. V, the numeric method from Section VIA, and the simulation method from Section VIB, are given in Figs. 3 and 4. The numerical and simulation methods agree exactly. The values of $\sigma_{v} /\langle v\rangle$ that will be considered in Section VII are shown in Table I and go up to about $50 \%$ for the most central collisions. Using the exact equations in Appendix A 2, points were plotted for these most central collisions in Fig. 3 at $v\{$ subEP $\} /\langle v\rangle=52.2 \%$. They agree exactly with the numeric and simulation methods, validating those methods. Thus one can see that the approximations of the analytic equations for the fluctuation dependence are less than about $0.5 \%$ for $v\{\mathrm{subEP}\} /\langle v\rangle$, and $1.0 \%$ for $v\{\mathrm{EP}\} /\langle v\rangle$. Since the $\delta$ values go up to about $20 \times 10^{-4}$ the approximations of the analytic equations for the nonflow dependence in Fig. 4 for $v\{\mathrm{EP}\} / v$ and $v\{\operatorname{subEP}\} / v$ are very small.

\section{APPLICATION TO DATA}

So far the equations have used generic fluctuation and nonflow parameters. To apply the equations to data we now assume that the fluctuations arise from participant eccentricity fluctuations and that the nonflow is related to the elliptic flow in $\mathrm{p}+\mathrm{p}$ collisions scaled by the number of participants. Thus to apply the analytic equations in Sec. $\mathrm{V}$ to extract $\langle v\rangle$ in the participant plane from experimental data, we have assumed that the fluctuations in $v$ have the same fractional width as the fluctuations of the participant eccentricity:

$$
\sigma_{v}=\frac{\sigma_{\varepsilon}}{\langle\varepsilon\rangle}\langle v\rangle
$$

Using this equation, Eq. (29) for $v\{2\}$ can be solved as

$$
\langle v\rangle=\sqrt{\left(v\{2\}^{2}-\delta\right) /\left(1+\left(\sigma_{\varepsilon} /\langle\varepsilon\rangle\right)^{2}\right)}
$$

and Eq. (30) for $v\{4\}$ as

$$
\langle v\rangle=v\{4\} /\left(1+\sigma_{\varepsilon} /\langle\varepsilon\rangle\right) .
$$

Because $\langle v\rangle$ appears in Eq. (8) for $\chi$, Eqs. (31) and (32) have to be solved by iteration.

\section{A. Glauber fluctuations}

A nucleon Monte-Carlo Glauber calculation was used to calculate the fractional standard deviation of $\varepsilon_{\text {part }}[21]$. The $\langle v\rangle$ values were calculated from $v\{2\}$ using Eq. (38) with $\delta=0$. Assuming Bessel-Gaussians, the resulting distributions are shown in Fig. 5.

For the nonflow contribution we have taken the value from proton-proton collisions and scaled it down by the number of participants. The value of $\delta_{p p}$ was obtained by integrating the minimum bias $\mathrm{p}+\mathrm{p}$ curves of Ref. [22], Fig. 1, and it was found that $\delta_{p p}=0.0145$ [23]. Thus for nonflow as a function of centrality we assume

$$
\delta=\delta_{p p} 2 / N_{\text {part }}
$$

knowing that in a $\mathrm{p}+\mathrm{p}$ collision there are two participants. One could also scale with $1 /$ multiplicity. Doing that we get as good results as shown below, but because 


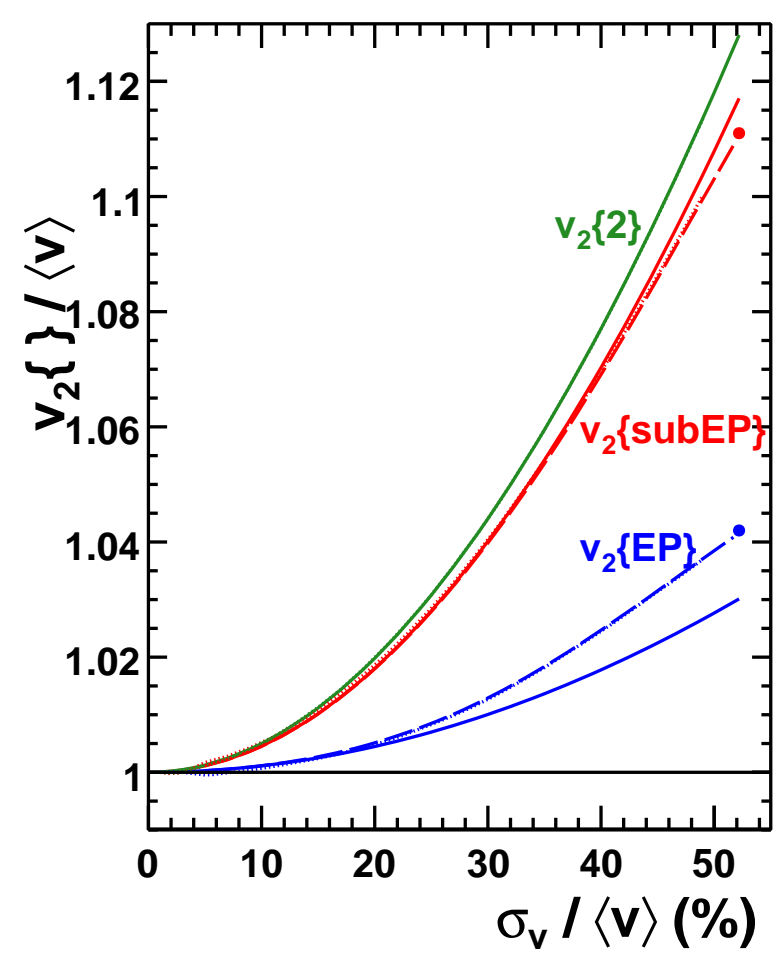

FIG. 3: (Color online) Various $v\{\}$ values as a function of the the magnitude of the fluctuations calculated for $\langle v\rangle=0.06$ with a full event multiplicity $=400$ and $\delta=0$. The solid curves are the analytic formulas. The points at $\sigma_{v} /\langle v\rangle=$ $52.2 \%$ are exact analytic calculations for zero impact parameter collisions. The dotted curves are from simulations. The dashed curves are the numerical integrations. The dotted curve for $v\{2\}$ is just under the solid curve. The dashed and dotted curves for $v\{\operatorname{subEP}\}$ and $v\{\mathrm{EP}\}$ coincide. Central collisions have large values of $\sigma_{v} /\langle v\rangle$.

multiplicity depends on acceptance, an extra parameter is needed.

The published STAR data $[2,24]$ for the various methods are shown in Fig. 6. The upper lines are from "twoparticle" correlation methods, and the lower line from a multiparticle correlation method. The lower line values for $v_{2}\{L Y Z\}$ are thought to be in the reaction plane, if the fluctuations are Gaussian [7]. The line for $v_{2}\{$ etaSub $\}$ is somewhat low for peripheral collisions because the gap in pseudorapidity reduces short-range nonflow correlations. Particularly puzzling is why the $v_{2}\{\mathrm{EP}\}$ line is lower than the other two-particle methods.

Correcting to $\langle v\rangle$ in the participant plane was done using Eq. (29) for $v_{2}\{2\}$, Eq. (30) for $v_{2}\{$ LYZ $\}$, Eq. (31) for $v_{2}\{\mathrm{EP}\}$, and Eq. (32) for $v_{2}\{\operatorname{ranSub}\}$ and $v_{2}\{$ etaSub $\}$. The results are shown in Fig. 7 . Since $v_{2}\{$ etaSub $\}$ is less affected by nonflow, the value of $\delta_{p p}$ used for it was multiplied by 0.5 . In Fig. 7 the convergence of the twoparticle, full event plane, and multiparticle results to one

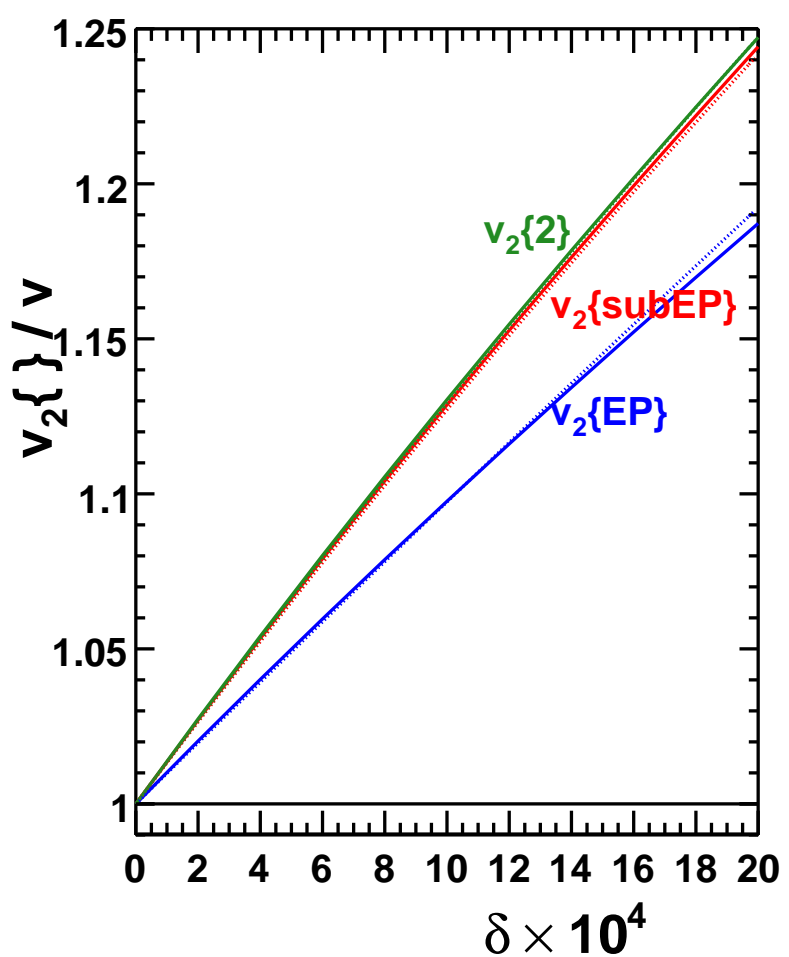

FIG. 4: (Color online) Various $v\{\}$ values as a function of the the magnitude of nonflow calculated for $\langle v\rangle=0.06$ with a full event multiplicity $=200$ and $\sigma_{v}=0$. The solid curves are the analytic formulas. The dotted curves are from simulations. The dotted curve for $v\{2\}$ is just under the solid curve. Peripheral collisions have large values of $\delta$.

locus in the participant plane is remarkable. Even the shape of the $v_{2}\{$ etaSub $\}$ curve has changed to match the others with only one additional parameter. Previously we took the spread in the values in Fig. 6 as an estimate of the systematic uncertainty.

\section{B. CGC fluctuations}

To see how sensitive the convergence of the different methods is to our assumptions for $\delta$ and $\sigma_{v}$, we also tried using fluctuations in $\varepsilon_{\text {part }}$ from the Color Glass Condensate (CGC) model [25]. In this model $\sigma_{\varepsilon} /\left\langle\varepsilon_{\text {part }}\right\rangle$ is roughly $30 \%$ smaller, mainly because $\left\langle\varepsilon_{\text {part }}\right\rangle$ is larger. Convergence of the methods was not obtained because the values of $\sigma_{\text {tot }}^{2} \equiv \delta_{2}+2 \sigma_{v 2}^{2}$ were too small. However, because of hard scattering one might argue that for nonflow the number of binary collisions is more important than the number of participants. In fact, raising $\delta$ by 


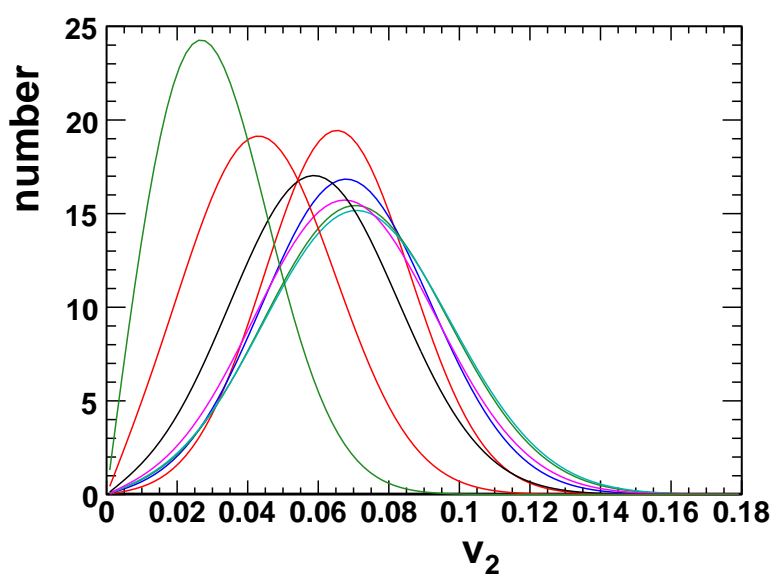

FIG. 5: (Color online) Bessel-Gaussian fluctuation distributions of $v$ assuming the same fractional width as $\varepsilon_{\text {part }}$ from Monte-Carlo Glauber calculations [21]. The different curves correspond to the first eight centrality bins of STAR for $\sqrt{\mathrm{s}_{\mathrm{NN}}}=200 \mathrm{GeV} \mathrm{Au}+\mathrm{Au}$. See Table I.

weighting with the number of binary collisions over the number of participants produced a slight over correction. However, adjusting $\delta$ to be $70 \%$ weighted with $N_{\text {bin }} / N_{\text {part }}$ and the rest just scaled with $1 / N_{\text {part }}$ brought $\sigma_{\text {tot }}$ down and produced reasonable convergence:

$$
\delta=\delta_{p p} 2\left[\left(x 2 N_{\text {bin }} / N_{\text {part }}\right)+(1-x)\right] / N_{\text {part }}
$$

with $x=0.7$. This assumes that for $\mathrm{p}+\mathrm{p}$ collisions, the number of participants is two and the number of binary interactions is one. Nonflow for $v_{2}\{$ etaSub $\}$ was reduced by multiplying by 0.7 . With these assumptions the results are shown in Fig. 8 in the participant plane. The convergence of the methods is also good.

\section{Reaction plane and parameters}

Using Eq. (13) and noting that $\langle v\rangle=v_{\mathrm{PP}}$ and $v\{4\} \simeq$ $v_{\mathrm{RP}}$, the reaction plane values can be obtained from the participant plane values by [7]:

$$
v_{\mathrm{PP}}^{2} \simeq v_{\mathrm{RP}}^{2}+\sigma_{v}^{2},
$$

and, by using Eq. (37)

$$
v_{\mathrm{RP}}=v_{\mathrm{PP}} \sqrt{1-\left(\sigma_{\varepsilon} /\langle\varepsilon\rangle\right)^{2}} .
$$

With this equation the values from Figs. 7 and 8 have been corrected to the reaction plane in Fig. 9. Thus, our two reasonable sets of assumptions about nonflow and fluctuations are not unique. At mid-centrality there is not much difference, but the graph illustrates the dependence on the systematic uncertainties in the assumptions that produce the corrections. $v_{2}$ in the reaction plane should go to zero at zero impact parameter. However, the first point in the graph is for $0-5 \%$ centrality and there is some smearing in determining the centrality from the experimental multiplicity. Also, because of ground state deformation of the Au nuclei, there could be some elliptic flow even at zero impact parameter [26].

The parameters used are shown in Fig. 10 and in Table I. The convergence depends on $\sigma_{\text {tot }}$, and is thus fixed from the experimental data by Eq. (33 top). It is about the same for the two sets of assumptions for centralities from $7.5 \%$ to $50 \%$. However, the two assumptions differ in the proportion of $\sigma_{v 2}$ and $\delta_{2}$ in $\sigma_{\text {tot }}$, and outside of this range the different assumptions give somewhat different results. We also tried the extreme assumption of no fluctuations and calculated $\delta_{2}$ at each centrality from $v_{2}\{2\}^{2}-v_{2}\{4\}^{2}$ as is indicated in Eq. (33 top) for $\sigma_{v}=0$. The convergence of the methods for centralities from $7.5 \%$ to $50 \%$ was good since $v_{2}\{2\}$ and $v_{2}\{4\}$ are forced together. However, for peripheral collisions there was less convergence amongst the other values than shown in Fig. 9, and these values were lower than the other two sets of curves. As $\sigma_{v 2}$ decreases from Glauber to CGC to zero, the more peripheral points decrease. That is because as $\sigma_{v 2}$ decreases, $\delta_{2}$ must increase to compensate, and the more peripheral bins are most affected by $\delta_{2}$. Although we can not rule out this nofluctuation assumption, the convergence of the methods is not as good as the other two cases.

\section{SUMMARY}

We have shown how the various experimental measures of elliptic flow are affected by fluctuations and nonflow, and derived analytic equations which are leading order in $\sigma_{v}^{2}$ and $\delta$. For $v\{\operatorname{subEP}\}$ and $v\{\mathrm{EP}\}$ we have shown how the analytic values for fluctuations differ from simulations and a numerical integration of the distribution of $v$. We have transformed published data to the participant plane and then to the reaction plane using reasonable assumptions for fluctuations and nonflow. The convergence of the various experimental measurements is remarkable. We have shown this for two sets of assumptions, showing how the values depend on these assumptions. The convergence of the methods essentially fixes the value of $\sigma_{\text {tot }}$ from experimental data, but the separation into fluctuation and nonflow parts is not unique. For that separation, better results for multiparticle correlations are needed.

This procedure could also be applied to differential flow. Probably the relative fluctuations $\sigma_{v} /\langle v\rangle$, but not the nonflow, should be independent of pseudorapidity and transverse momentum. The nonflow as a function of $p_{T}$ might be obtained from $\mathrm{p}+\mathrm{p}$ collisions as was done here for the integrated flow. 


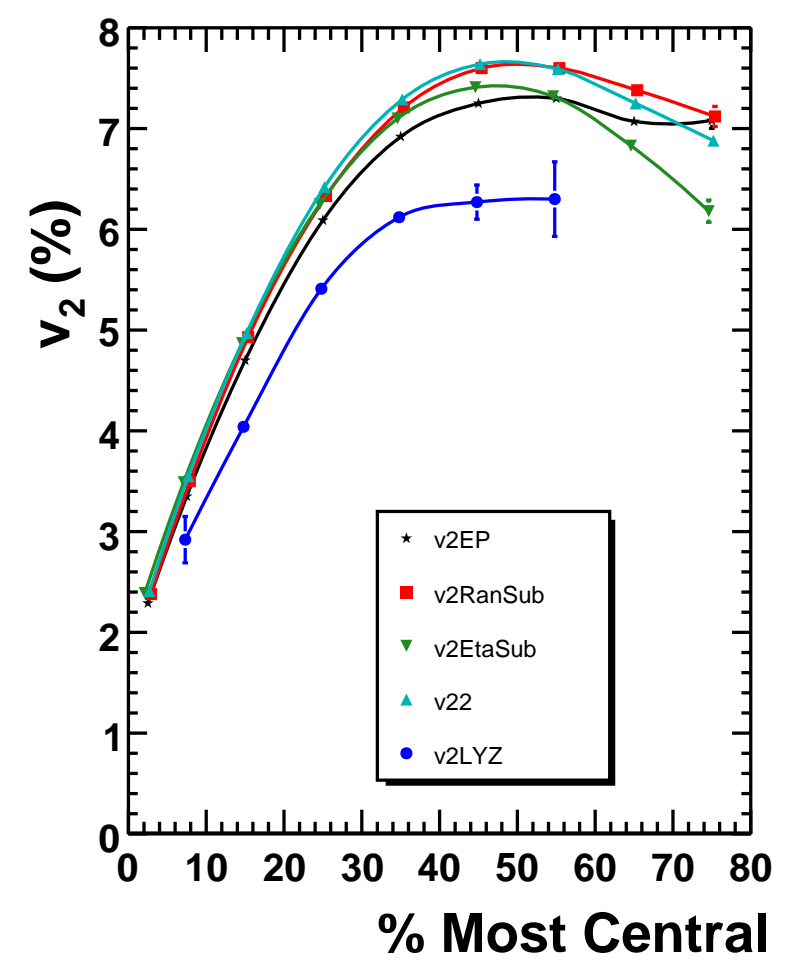

FIG. 6: (Color online) The values of $v_{2}$ from various analysis methods vs centrality. Both the upper lines [2] and the lower line [24] are STAR data.

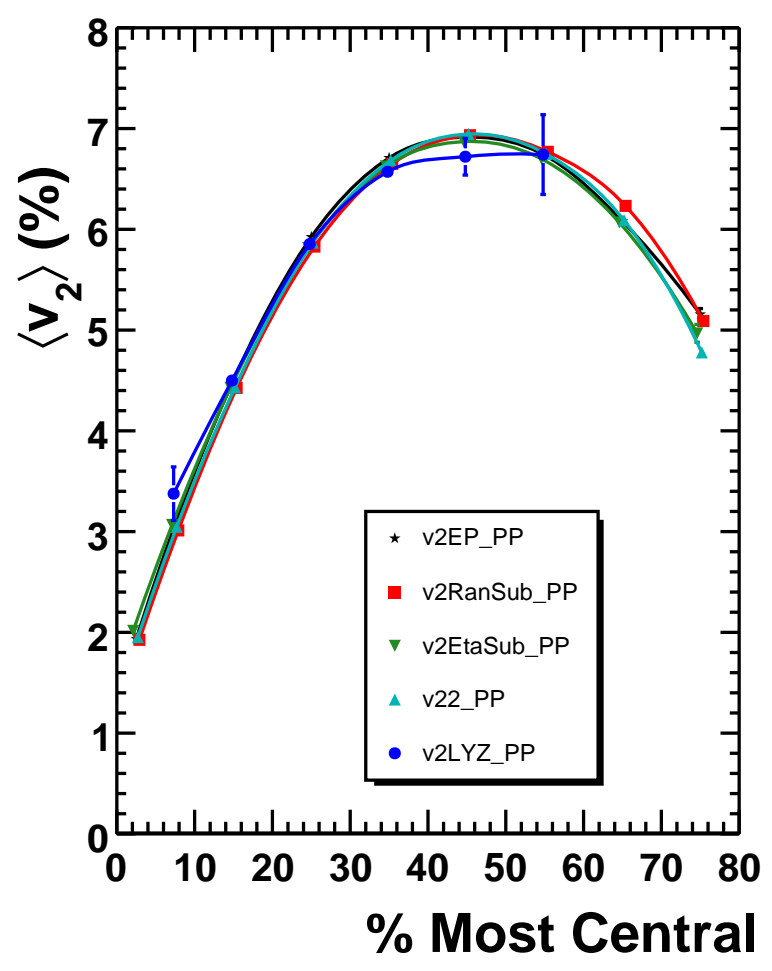

FIG. 7: (Color online) Shown are the data from Fig. 6 corrected to $\left\langle v_{2}\right\rangle$ in the participant plane.

\begin{tabular}{rrr|rrrrrrr}
\hline \hline \multicolumn{1}{c}{ Glauber } & \multicolumn{4}{c}{ CGC } \\
bin & centrality & mult & $\sigma_{\varepsilon} /\langle\varepsilon\rangle$ & $N_{\text {part }}$ & $\sigma_{\text {tot }}^{2}$ & $\sigma_{\varepsilon} /\langle\varepsilon\rangle$ & $N_{\text {bin }}$ & $\sigma_{\text {tot }}^{2}$ \\
\hline 9 & $0-05 \%$ & 961 & $55.5 \%$ & 352 & 4.05 & $48.9 \%$ & 1049 & 6.19 \\
8 & $05-10 \%$ & 819 & $50.2 \%$ & 298 & 6.63 & $32.7 \%$ & 825 & 7.25 \\
7 & $10-20 \%$ & 651 & $44.0 \%$ & 232 & 9.80 & $31.7 \%$ & 587 & 9.24 \\
6 & $20-30 \%$ & 468 & $38.2 \%$ & 165 & 12.6 & $28.1 \%$ & 364 & 11.8 \\
5 & $30-40 \%$ & 323 & $36.4 \%$ & 114 & 15.2 & $28.3 \%$ & 216 & 15.2 \\
4 & $40-50 \%$ & 214 & $36.0 \%$ & 75 & 17.5 & $30.1 \%$ & 120 & 19.3 \\
3 & $50-60 \%$ & 134 & $35.6 \%$ & 46 & 19.8 & $31.7 \%$ & 61 & 24.3 \\
2 & $60-70 \%$ & 76 & $34.1 \%$ & 26 & 22.8 & $32.0 \%$ & 28 & 30.4 \\
1 & $70-80 \%$ & 38 & $31.0 \%$ & 13 & 31.9 & $32.0 \%$ & 11 & 43.4 \\
\hline \hline
\end{tabular}

TABLE I: For each centrality are shown the full event multiplicity [2], the standard deviation of Monte-Carlo Glauber $\varepsilon_{\text {part }}$ in percent of the mean [21], the number of participants [2], Glauber $\sigma_{\text {tot }}^{2} \times 10^{4}$ as calculated here, the standard deviation of CGC $\varepsilon_{\text {part }}$ in percent of the mean [25], the number of binary collisions [2], and CGC $\sigma_{\text {tot }}^{2} \times 10^{4}$ as calculated here. The values of $\delta_{2}$ are given by Eq. (40) for the Glauber model and Eq. (41) for the CGC model.

\section{ACKNOWLEDGMENTS}

We thank the authors of Ref. [19] and Constantin Loizides for permision to use Fig. 1. For discussions we thank Hiroshi Masui, Aihong Tang and Paul Sorensen. This work was supported in part by the HENP Divisions of the Office of Science of the US Department of Energy under Contract Numbers DE-AC02-05CH11231 and DEFG02-92ER40713.
Appendix A: Effects of fluctuations on $v\{\mathrm{EP}\}$

We derive the difference $\delta v$ between $v\{\mathrm{EP}\}$ and $\langle v\rangle$ due to fluctuations, to leading order in $\sigma_{v}^{2}$, assuming that nonflow effects are negligible. Flow fluctuations modify both the numerator and the denominator of Eq. (4). 


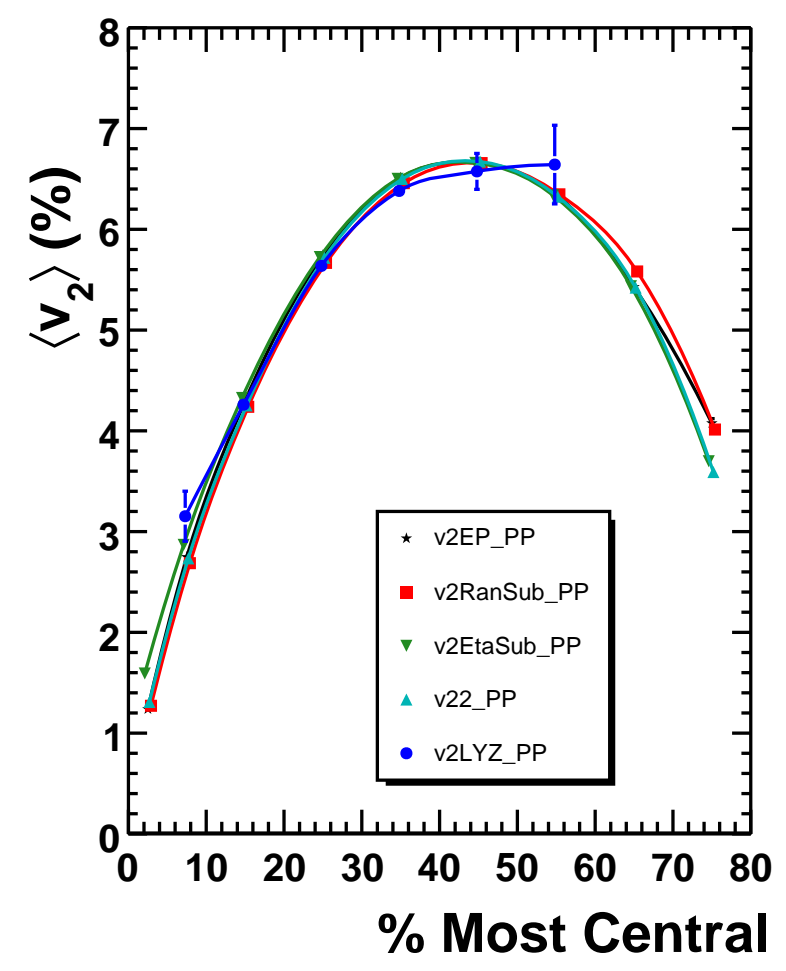

FIG. 8: (Color online) Shown are the data from Fig. 6 corrected to $\left\langle v_{2}\right\rangle$ in the participant plane using CGC fluctuations and nonflow partly weighted with $N_{\text {bin }}$.

\section{Small fluctuations}

For small fluctuations, the relative change of $v\{\mathrm{EP}\}$ is obtained by taking the logarithm of Eq. (4) and differentiating

$$
\frac{\delta v}{\langle v\rangle} \equiv \frac{v\{\mathrm{EP}\}-\langle v\rangle}{\langle v\rangle}=\frac{\delta\left\langle\cos \left(\phi-\Psi_{R}\right)\right\rangle}{\left\langle\cos \left(\phi-\Psi_{R}\right)\right\rangle}-\frac{\delta R}{R} .
$$

The first term on the right-hand side is the contribution of fluctuations to the correlation with the event plane, the second term is the contribution of fluctuations to the resolution. We evaluate these contributions in turn.

The resolution parameter $\chi$ in Eq. (7) is proportional to the flow $v$. If the analysis is done with unit weights as in Eq. (3), then $\chi=v \sqrt{N}$, where $N$ is the number of particles in the event plane. More generally, we write $\chi=$ $r v$, where $r$ is a parameter depending on the details of the analysis. For a given value of $v,\left\langle\cos \left(\phi-\Psi_{R}\right)\right\rangle=v \mathcal{R}(r v)$. If $v$ fluctuates, one must average this quantity over the fluctuations of $v$. Using Eq. (11) with $f(v)=v \mathcal{R}(r v)$, the relative change due to fluctuations is

$$
\frac{\delta\left\langle\cos \left(\phi-\Psi_{R}\right)\right\rangle}{\left\langle\cos \left(\phi-\Psi_{R}\right)\right\rangle}=\frac{\sigma_{v}^{2}}{2} \frac{\frac{d^{2}}{d v^{2}}(v \mathcal{R}(r v))}{\langle v\rangle \mathcal{R}(r\langle v\rangle)}
$$

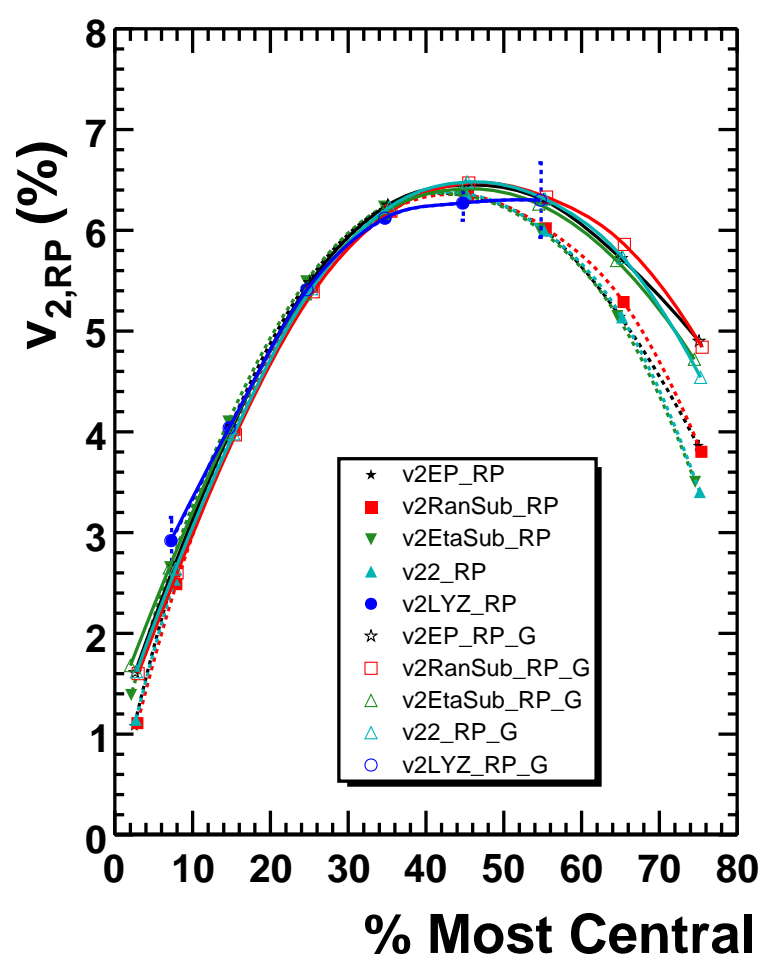

FIG. 9: (Color online) Shown are the data from Figs. 7 and 8 corrected to $\left\langle v_{2}\right\rangle$ in the reaction plane. The solid lines are for the Glauber model of fluctuations and the dashed lines for the CGC model of fluctuations.

$$
=\frac{\sigma_{v}^{2}}{2\langle v\rangle^{2}} \frac{\chi \frac{d^{2}}{d \chi^{2}}(\chi \mathcal{R}(\chi))}{\mathcal{R}(\chi)} .
$$

In the second equality, $\chi \equiv r\langle v\rangle$ denotes the resolution parameter associated with $\langle v\rangle$. Using Eq. (7), one obtains after some algebra:

$$
\frac{\delta\left\langle\cos \left(\phi-\Psi_{R}\right)\right\rangle}{\left\langle\cos \left(\phi-\Psi_{R}\right)\right\rangle}=\frac{\sigma_{v}^{2}}{2\langle v\rangle^{2}}\left(1+\frac{I_{0}-I_{1}}{I_{0}+I_{1}}\left(1-2 \chi^{2}\right)\right)(
$$

and $I_{0,1}$ is a shorthand notation for $I_{0,1}\left(\chi^{2} / 2\right)$.

We now evaluate the second term in Eq. (A1), namely, the shift in the resolution due to fluctuation. In order to estimate the resolution experimentally, one correlates two subevents $A$ and $B$. In the absence of fluctuations, $\left\langle\cos \left(\Psi_{A}-\Psi_{B}\right)\right\rangle=\mathcal{R}\left(\chi_{s}\right)^{2}$, where $\chi_{s}$ is the resolution parameter of one subevent. With unit weights, $\chi_{s}=$ $v \sqrt{N_{s}}$, where $N_{s}$ is the number of particles in a subevent. More generally, one can write $\chi_{s}=r_{s} v$, where $r_{s}$ is a parameter which depends on the details of the analysis. The modification of the correlation due to fluctuations is evaluated using Eq. (11), with $f(v)=\mathcal{R}\left(r_{s} v\right)^{2}$ :

$$
\frac{\delta\left\langle\cos \left(\Psi_{A}-\Psi_{B}\right)\right\rangle}{\left\langle\cos \left(\Psi_{A}-\Psi_{B}\right)\right\rangle}=\frac{\sigma_{v}^{2}}{2} \frac{\frac{d^{2}}{d v^{2}}\left(\mathcal{R}\left(r_{s} v\right)^{2}\right)}{\mathcal{R}\left(r_{s}\langle v\rangle\right)^{2}}
$$




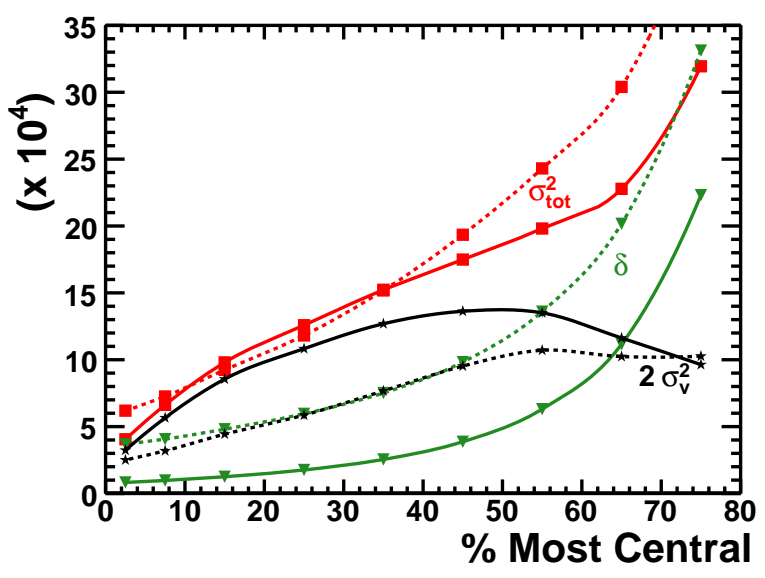

FIG. 10: (Color online) The nonflow and fluctuation parameters derived from assumptions in the text, which were used to make the corrections of the various $v_{2}$ values to $\left\langle v_{2}\right\rangle$ in the participant plane and then to the reaction plane. $\sigma_{\text {tot }}^{2} \equiv \delta_{2}+2 \sigma_{v 2}^{2}$. See Table I. The solid lines are for the Glauber model with $1 / N_{\text {part }}$ scaling of $\delta$, and the dashed lines are for the CGC model with the addition of partial binary weighting for $\delta$.

$$
=\frac{\sigma_{v}^{2}}{2\langle v\rangle^{2}} \frac{\chi_{s}^{2} \frac{d^{2}}{d \chi_{s}^{2}}\left(\mathcal{R}\left(\chi_{s}\right)^{2}\right)}{\mathcal{R}\left(\chi_{s}\right)^{2}} .
$$

In the second equality, $\chi_{s} \equiv r_{s}\langle v\rangle$ denotes the mean subevent resolution parameter.

The resolution parameter of the subevent is determined experimentally by solving Eq. (6). We denote by $\chi_{s}^{\exp }$ the solution of this equation, and by $\delta \chi_{s}$ the shift due to fluctuations: $\chi_{s}^{\exp }=\chi_{s}+\delta \chi_{s}$. Differentiating Eq. (6), we obtain, to leading order in $\delta \chi_{s}$ :

$$
\frac{\mathcal{R}^{\prime}\left(\chi_{s}\right) \delta \chi_{s}}{\mathcal{R}\left(\chi_{s}\right)}=\frac{1}{2} \frac{\delta\left\langle\cos \left(\Psi_{A}-\Psi_{B}\right)\right\rangle}{\left\langle\cos \left(\Psi_{A}-\Psi_{B}\right)\right\rangle}
$$

Inserting Eq. (A4), one obtains

$$
\frac{\delta \chi_{s}}{\chi_{s}}=\frac{\sigma_{v}^{2}}{2\langle v\rangle^{2}} \frac{\chi_{s}}{2 \mathcal{R}\left(\chi_{s}\right) \mathcal{R}^{\prime}\left(\chi_{s}\right)} \frac{d^{2}}{d \chi_{s}^{2}}\left(\mathcal{R}\left(\chi_{s}\right)^{2}\right) .
$$

Using Eq. (7), one obtains after some algebra:

$$
\frac{\delta \chi_{s}}{\chi_{s}}=\frac{\sigma_{v}^{2}}{2\langle v\rangle^{2}}\left(-2 \chi_{s}^{2}+1+\frac{4 i_{1}^{2}}{i_{0}^{2}-i_{1}^{2}}\right) .
$$

where $i_{0,1}$ is a shorthand notation for $I_{0,1}\left(\chi_{s}^{2} / 2\right)$. The resolution parameter of the whole event, $\chi^{\exp }$, is defined from the subevent resolution $\chi_{s}^{\exp }$ by

$$
\chi^{\exp } \equiv \sqrt{N / N_{s}} \chi_{s}^{\exp }=\left(r / r_{s}\right) \chi_{s}^{\exp }
$$

Writing $\chi^{\exp }=\chi+\delta \chi$, where $\delta \chi$ is the shift due to fluctuations, the resulting change $\delta R$ in the resolution of the whole event is given by Eq. (9):

$$
\frac{\delta R}{R}=\frac{\chi \mathcal{R}^{\prime}(\chi)}{\mathcal{R}(\chi)} \frac{\delta \chi}{\chi}=\frac{\chi \mathcal{R}^{\prime}(\chi)}{\mathcal{R}(\chi)} \frac{\delta \chi_{s}}{\chi_{s}} .
$$

Using Eq. (7), we obtain

$$
\frac{\chi \mathcal{R}^{\prime}(\chi)}{\mathcal{R}(\chi)}=\frac{I_{0}-I_{1}}{I_{0}+I_{1}}
$$

Inserting Eqs. (A7) and (A10) into (A9), we obtain

$$
\frac{\delta R}{R}=-\frac{I_{0}-I_{1}}{I_{0}+I_{1}}\left(-2 \chi_{s}^{2}+1+\frac{4 i_{1}^{2}}{i_{0}^{2}-i_{1}^{2}}\right) \frac{\sigma_{v}^{2}}{2\langle v\rangle^{2}} .
$$

Inserting Eqs. (A3) and (A11) into Eq. (A1), one obtains $\delta v /\langle v\rangle$. Finally, using

$$
v\{\mathrm{EP}\}^{2}=(\langle v\rangle+\delta v)^{2} \simeq\langle v\rangle^{2}+2\langle v\rangle^{2} \frac{\delta v}{\langle v\rangle},
$$

one obtains Eq. (22).

\section{Central collisions}

Flow fluctuations are largest, in relative magnitude, for central collisions. In this section, we derive exact formulas for the effect of fluctuations on $v\{\mathrm{EP}\}$ and $v\{\mathrm{subEP}\}$ in central collisions. By comparing these exact results with the approximate formulas derived above for small fluctuations, we will be able to assess the accuracy of the small fluctuation approximations.

We assume that flow fluctuations result from Gaussian eccentricity fluctuations [7]. The flow is given by $v=$ $\sqrt{v_{x}^{2}+v_{y}^{2}}$, where the distribution of $\mathbf{v}=\left(v_{x}, v_{y}\right)$ is a two-dimensional Gaussian

$$
\frac{d n}{d v_{x} d v_{y}}=\frac{1}{2 \pi \sigma_{0}^{2}} \exp \left(-\frac{\left(v_{x}-v_{0}\right)^{2}+v_{y}^{2}}{2 \sigma_{0}^{2}}\right) .
$$

Integrating over the azimuthal angle of $\mathbf{v}$, one recovers the Bessel-Gaussian distribution (34). From now on, we assume $v_{0}=0$, as expected by symmetry for central collisions with no flow. Then, the first two moments of the distribution are $\langle v\rangle=\sqrt{\pi / 2} \sigma_{0}$ and $\left\langle v^{2}\right\rangle=2 \sigma_{0}^{2}$. For a given value of $\mathbf{v}$, the distribution of the flow vector of a subevent (A or B) is also a Gaussian centered around the direction of $\mathbf{v}[27,28]$ :

$$
p_{\mathbf{v}}\left(\mathbf{q}_{A, B}\right)=\frac{1}{\pi} \exp \left(-\left(\mathbf{q}_{A, B}-\mathbf{v} \sqrt{N / 2}\right)^{2}\right) .
$$

The distribution of the flow vector of the whole event is given by a similar equation, with $N$ instead of $N / 2$. A factor $N$ comes from having $N$ particles in the event plane, and a factor $1 / \sqrt{N}$ comes from the definition of the flow vector, Eq. (3). The resolution $\mathcal{R}(\chi)$ in Eq. (7) is obtained by computing the average value of $\cos \Delta \Phi_{R} \equiv$ $q_{x} / q$ with this distribution. When $v$ fluctuates, it is in fact easier to integrate first over $v$, then over $q$. One thus obtains the numerator of Eq. (35)

$$
\langle v \mathcal{R}(v \sqrt{N / 2})\rangle=\frac{\chi_{s}}{\sqrt{(\pi / 4)+\chi_{s}^{2}}}\langle v\rangle,
$$


where $\chi_{s} \equiv\langle v\rangle \sqrt{N / 2}$ is the average resolution parameter of a subevent. The numerator of Eq. (36) is given by a similar equation, with $\chi_{s}$ replaced by $\chi=\langle v\rangle \sqrt{N}$.

We now evaluate the correlation between subevents. Let $\mathbf{q}_{A}$ and $\mathbf{q}_{B}$ denote the flow vectors of subevents $A$ and $B$. Neglecting nonflow correlations, the joint probability distribution of $\mathbf{q}_{A}$ and $\mathbf{q}_{B}$ is $p_{\mathbf{v}}\left(\mathbf{q}_{A}\right) p_{\mathbf{v}}\left(\mathbf{q}_{B}\right)$ for a given flow $\mathbf{v}$. Integrating over $\mathbf{v}$, one obtains the following probability distribution for $\left(\mathbf{q}_{A}, \mathbf{q}_{B}\right)$ :

$$
\begin{aligned}
& p\left(\mathbf{q}_{A}, \mathbf{q}_{B}\right)=\frac{1}{\pi^{2}\left(1-C^{2}\right)} \\
& \quad \times \exp \left(-\frac{q_{A}^{2}+q_{B}^{2}-2 C \mathbf{q}_{A} \cdot \mathbf{q}_{B}}{1-C^{2}}\right),
\end{aligned}
$$

where

$$
C \equiv \frac{N \sigma_{0}^{2}}{1+N \sigma_{0}^{2}}
$$

is the linear correlation between $\mathbf{q}_{A}$ and $\mathbf{q}_{B}$. The probability distribution (A16) is a correlated Gaussian distribution, which is formally identical to the distribution in the presence of nonflow effects $[29,30]$. The relative angle between subevents, $\Delta \Phi$, is given by $\cos \Delta \Phi=$ $\mathbf{q}_{A} \cdot \mathbf{q}_{B} /\left(q_{A} q_{B}\right)$. Integrating over $\mathbf{q}_{A}$ and $\mathbf{q}_{B}$, one obtains after some algebra:

$$
\left\langle[\mathcal{R}(v \sqrt{N / 2})]^{2}\right\rangle=\langle\cos \Delta \Phi\rangle=\frac{E\left(C^{2}\right)-\left(1-C^{2}\right) K\left(C^{2}\right)}{C},
$$

where $K$ and $E$ are complete elliptic integrals of the first and second kind. $\langle\cos \Delta \Phi\rangle$ is approximately $(\pi / 4) C$ for $C \ll 1$, and is equal to 1 for $C=1$. The exact values of $v\{$ subEP $\}$ and $v\{\mathrm{EP}\}$ are obtained by inserting Eqs. (A15) and (A18) into Eqs. (35) and (36). The ratio of these exact results to the approximate expressions Eqs. (21) and (22) is plotted in Fig. 2.

\section{Appendix B: Effects of nonflow correlations on $v\{\mathrm{EP}\}$}

The nonflow correlation is denoted by $\delta$ in Eq. (24). In this Appendix, we denote it by $\delta_{\text {nf }}$ to avoid ambiguity, while $\delta X$ denotes the small change of an observable $X$ due to nonflow correlations. We derive the expression of $v\{\mathrm{EP}\}$ to leading order in $\delta_{\mathrm{nf}}$, neglecting flow fluctuations.

In the same way as fluctuations, nonflow effects contribute to both the numerator and denominator of Eq. (4): Eq. (A1) still holds, except that the shift is due to nonflow instead of fluctuations. Nonflow effects give a direct contribution to the correlation between the particle and the event plane, and to the correlation between subevents. In addition, nonflow effects modify the distribution of the flow vector, which induces a change in the resolution parameter. We evaluate all these nonflow contributions separately when the flow vector is defined with unit weights, as in Eq. (3). In practice, the analysis is often done with $p_{T}$ weights to increase the resolution. This case is more complex and will be discussed at the end.

\section{Correction to the resolution parameter}

The normalized probability distribution of the flow vector, defined by Eq. (3), is Gaussian:

$$
p(\mathbf{q})=\frac{1}{\pi \sigma^{2}} \exp \left(-\frac{\left(\mathbf{q}-v \sqrt{N} \mathbf{e}_{x}\right)^{2}}{\sigma^{2}}\right)
$$

$\mathbf{e}_{x}$ is the unit vector along the true reaction plane, chosen as the $x$ axis. In the absence of nonflow effects, $\sigma=1$ due to the normalization factor $1 / \sqrt{N}$ in Eq. (3), and Eq. (B1) reduces to

$$
\begin{aligned}
p(\mathbf{q}) & =\frac{d N}{q d q d \Psi_{R}} \\
& =\frac{1}{\pi} \exp \left(-q^{2}-\chi^{2}+2 q \chi \cos \Psi_{R}\right) .
\end{aligned}
$$

Nonflow effects modify $\sigma$. Since the flow vector $\mathbf{q}$ in Eq. (3) involves $N$ particles, the average value of $q^{2}$ involves $N^{2}$ correlated pairs. These pairs have nonflow correlations, defined by Eq. (24). With the $1 / \sqrt{N}$ normalization factor in Eq. (3), one obtains

$$
\sigma^{2}=1+N \delta_{\mathrm{nf}}
$$

This change in $\sigma$ induces a change in the resolution parameter [29]:

$$
\frac{\delta \chi}{\chi}=-\frac{\delta \sigma}{\sigma}=-\frac{N \delta_{\mathrm{nf}}}{2} .
$$

Similarly, the resolution parameter of subevents, $\chi_{s}$, is changed by the amount

$$
\frac{\delta \chi_{s}}{\chi_{s}}=-\frac{N_{s} \delta_{\mathrm{nf}}}{2}
$$

\section{Correlation with the event plane}

Without nonflow effects, the correlation between the particle and the event plane is

$$
\left\langle\cos \left(\phi-\Psi_{R}\right)\right\rangle=v \mathcal{R}(\chi)
$$

Nonflow effects modify this equation in two different ways: 1) the nonflow correlation between the particle and the event plane adds an extra term to the right-hand side; 2) $\chi$ is modified according to Eq. (B4).

We first evaluate the nonflow correlation between the particle and the event plane. Let $\mathbf{u} \equiv(\cos \phi, \sin \phi)$ denote the unit vector of the particle momentum. As 
shown in Ref. [29] in the case of momentum conservation, nonflow correlations between the flow vector and the particle amount to shifting the flow vector by a small amount proportional to $\mathbf{u}$. It can easily be shown that the shift is $\delta_{\mathrm{nf}} \sqrt{N} \mathbf{u}$, where a factor $N$ comes from having $N$ particles in the event plane, and a factor $1 / \sqrt{N}$ comes from the definition of $\mathbf{q}$, Eq. (3). The correlation between the particle and the event plane can be written as $\cos \left(\phi-\Psi_{R}\right)=\mathbf{u} \cdot \mathbf{q} / q$. Shifting the flow vector and expanding to leading order in $\delta_{\mathrm{nf}}$, the resulting contribution to $\cos \left(\phi-\Psi_{R}\right)$ is

$$
\begin{aligned}
\delta \cos \left(\phi-\Psi_{R}\right) & =\frac{\sqrt{N} \delta_{\mathrm{nf}}}{q}\left(1-\frac{(\mathbf{u} \cdot \mathbf{q})^{2}}{q^{2}}\right) \\
& =\frac{\sqrt{N} \delta_{\mathrm{nf}}}{q} \sin ^{2}\left(\phi-\Psi_{R}\right)
\end{aligned}
$$

Averaging over events, $\sin ^{2}\left(\phi-\Psi_{R}\right)$ gives $1 / 2$. The average value of $1 / q$ is computed using Eq. (B2):

$$
\left\langle\frac{1}{q}\right\rangle=\int \frac{1}{q} \frac{d N}{q d q d \Psi_{R}} q d q d \Psi_{R}=\frac{\sqrt{\pi}}{2} e^{-\chi^{2} / 2} 2 I_{0} .
$$

In order to obtain the relative change due to nonflow effects, we divide by Eq. (B6), where $\mathcal{R}(\chi)$ is given by Eq. (7), and $\chi=v \sqrt{N}$ :

$$
\frac{\delta\left\langle\cos \left(\phi-\Psi_{R}\right)\right\rangle}{\left\langle\cos \left(\phi-\Psi_{R}\right)\right\rangle}=\frac{2 I_{0}}{\left(I_{0}+I_{1}\right)} \frac{\delta_{\mathrm{nf}}}{2 v^{2}}=\left(1+\frac{I_{0}-I_{1}}{I_{0}+I_{1}}\right) \frac{\delta_{\mathrm{nf}}}{2 v^{2}}
$$

We now evaluate the second contribution, arising from the modification of $\chi$, Eq. (B4). The resulting change is

$$
\frac{\delta\left\langle\cos \left(\phi-\Psi_{R}\right)\right\rangle}{\left\langle\cos \left(\phi-\Psi_{R}\right)\right\rangle}=\frac{\delta \mathcal{R}}{\mathcal{R}}=\frac{\mathcal{R}^{\prime}(\chi)}{\mathcal{R}(\chi)} \delta \chi=-\frac{\chi \mathcal{R}^{\prime}(\chi)}{\mathcal{R}(\chi)} \frac{N \delta_{\mathrm{nf}}}{2} .
$$

Using Eq. (A10) and $N=\chi^{2} / v^{2}$, this becomes

$$
\frac{\delta\left\langle\cos \left(\phi-\Psi_{R}\right)\right\rangle}{\left\langle\cos \left(\phi-\Psi_{R}\right)\right\rangle}=-\frac{I_{0}-I_{1}}{I_{0}+I_{1}} \chi^{2} \frac{\delta_{\mathrm{nf}}}{2 v^{2}}
$$

Adding the contributions from Eqs. (B9) and (B11), we obtain the total nonflow contribution to the correlation between the particle and the event plane:

$$
\frac{\delta\left\langle\cos \left(\phi-\Psi_{R}\right)\right\rangle}{\left\langle\cos \left(\phi-\Psi_{R}\right)\right\rangle}=\left(1+\frac{\left(I_{0}-I_{1}\right)}{\left(I_{0}+I_{1}\right)}\left(1-\chi^{2}\right)\right) \frac{\delta_{\mathrm{nf}}}{2 v^{2}}
$$

\section{Resolution correction}

We now derive the modification of the resolution correction due to nonflow effects. As in Sec. B 2, there are two nonflow contributions: the first contribution is the nonflow correlation between the subevents; the second modification arises from the modification of the width of the flow vector distribution, Eq. (B5).

We first derive the correlation between subevents due to nonflow effects. Let $\mathbf{q}_{A}$ and $\mathbf{q}_{B}$ denote the flow vectors of subevents $A$ and $B$. The joint probability distribution of $\mathbf{q}_{A}$ and $\mathbf{q}_{B}$ is [29]:

$$
\frac{d N}{d^{2} \mathbf{q}_{A} d^{2} \mathbf{q}_{B}}=p\left(\mathbf{q}_{A}\right) p\left(\mathbf{q}_{B}\right)\left(1+2 N_{s} \delta_{\mathrm{nf}}\left(\mathbf{q}_{A}-\chi \mathbf{e}_{x}\right) \cdot\left(\mathbf{q}_{B}-\chi \mathbf{e}_{x}\right)\right),
$$

where $p\left(\mathbf{q}_{A}\right)$ is defined by Eq. (B2) (except that $\chi$ is replaced with $\chi_{s}$ ), and the term proportional to $\delta_{\text {nf }}$ is the nonflow correlation between subevents. The factor $N_{s}$ is due to the fact that the correlation is $N_{s}$ times stronger between subevents than between individual particles. One then computes $\left\langle\cos \left(\Psi_{A}-\Psi_{B}\right)\right\rangle$ with this probability distribution. The nonflow contribution reads:

$$
\delta\left\langle\cos \left(\Psi_{A}-\Psi_{B}\right)\right\rangle=2 N_{s} \delta_{\mathrm{nf}}\left(\left\langle\left(q \cos \Psi_{A}-\chi\right) \cos \Psi_{A}\right\rangle^{2}+\left\langle q \sin ^{2} \Psi_{A}\right\rangle^{2}\right),
$$

where angular brackets on the right-hand side denote average values, which are taken with the probability distribution Eq. (B2). These averages are easily evaluated:

$$
\left\langle\left(q \cos \Psi_{A}-\chi\right) \cos \Psi_{A}\right\rangle=\frac{\sqrt{\pi}}{2} e^{-\chi_{s}^{2} / 2} \frac{1}{2}\left(i_{0}-i_{1}\right),
$$

and

$$
\left\langle q \sin ^{2} \Psi_{A}\right\rangle=\frac{\sqrt{\pi}}{2} e^{-\chi_{s}^{2} / 2} \frac{1}{2}\left(i_{0}+i_{1}\right)
$$

One thus obtains

$$
\delta\left\langle\cos \left(\Psi_{A}-\Psi_{B}\right)\right\rangle=N_{s} \delta_{\mathrm{nf}}\left(\frac{\sqrt{\pi}}{2} e^{-\chi_{s}^{2} / 2}\right)^{2}\left(i_{0}^{2}+i_{1}^{2}\right) .
$$

In the absence of nonflow effects, $\left\langle\cos \left(\Psi_{A}-\Psi_{B}\right)\right\rangle=$ $\mathcal{R}\left(\chi_{s}\right)^{2}$, where $\mathcal{R}\left(\chi_{s}\right)$ is given by Eq. (7). This gives the relative variation

$$
\frac{\delta\left\langle\cos \left(\Psi_{A}-\Psi_{B}\right)\right\rangle}{\left\langle\cos \left(\Psi_{A}-\Psi_{B}\right)\right\rangle}=\frac{i_{0}^{2}+i_{1}^{2}}{\left(i_{0}+i_{1}\right)^{2}} \frac{\delta_{\mathrm{nf}}}{2 v^{2}},
$$

where we have used $N_{s} / \chi_{s}^{2}=1 / v^{2}$. Nonflow effects intro- 
duce a bias $\delta \chi_{s}$ in the estimate of $\chi_{s}$, the resolution parameter of the subevent. This bias is given by Eq. (A5):

$$
\frac{\delta \chi_{s}}{\chi_{s}}=\frac{\delta\left\langle\cos \left(\Psi_{A}-\Psi_{B}\right)\right\rangle}{\left\langle\cos \left(\Psi_{A}-\Psi_{B}\right)\right\rangle} \frac{\mathcal{R}\left(\chi_{s}\right)}{\chi_{s} \mathcal{R}^{\prime}\left(\chi_{s}\right)}=\frac{\left(i_{0}^{2}+i_{1}^{2}\right)}{\left(i_{0}^{2}-i_{1}^{2}\right)} \frac{\delta_{\mathrm{nf}}}{2 v^{2}},
$$

where we have used Eq. (A10), with $\chi_{s}$ instead of $\chi$. The second effect is the modification of $\chi_{s}$ from the increase of the width of the distribution of the flow vector, Eq. (B5). Writing $N_{s}=\chi_{s}^{2} / v^{2}$ and adding this contribution to Eq. (B19), we obtain

$$
\frac{\delta \chi_{s}}{\chi_{s}}=\left(\frac{i_{0}^{2}+i_{1}^{2}}{i_{0}^{2}-i_{1}^{2}}-\chi_{s}^{2}\right) \frac{\delta_{\mathrm{nf}}}{2 v^{2}}=\left(1-\chi_{s}^{2}+\frac{2 i_{1}^{2}}{i_{0}^{2}-i_{1}^{2}}\right) \frac{\delta_{\mathrm{nf}}}{2 v^{2}}
$$

The relative correction to the resolution is then given by Eqs. (A9) and (A10): Inserting Eq. (B20),we obtain

$$
\frac{\delta R}{R}=\frac{I_{0}-I_{1}}{I_{0}+I_{1}}\left(1-\chi_{s}^{2}+\frac{2 i_{1}^{2}}{i_{0}^{2}-i_{1}^{2}}\right) \frac{\delta_{\mathrm{nf}}}{2 v^{2}} .
$$

The relative change $\delta v / v$ is obtained from Eqs. (A1), using the results from Eqs. (B12) and (B21). Eq. (27) is finally obtained using Eq. (A12).

\section{Weights}

We finally discuss the case where the flow analysis is done with weights. This means that Eq. (3) is replaced with:

$$
\mathbf{q} \cos \Psi_{R}=\frac{\mathbf{Q}}{\sqrt{N}} \cos \Psi_{R}=\frac{1}{\sqrt{N}} \sum_{j=1}^{N} w_{j} \cos \phi_{j}
$$

[1] S. A. Voloshin, A. M. Poskanzer and R. Snellings, arXiv:0809.2949 [nucl-ex].

[2] J. Adams et al. [STAR Collaboration], Phys. Rev. C 72, 014904 (2005) [arXiv:nucl-ex/0409033].

[3] H. Song and U. W. Heinz, arXiv:0812.4274 [nucl-th].

[4] N. Borghini, P. M. Dinh and J. Y. Ollitrault, Phys. Rev. C 62, 034902 (2000) [arXiv:nucl-th/0004026].

[5] M. Miller and R. Snellings, arXiv:nucl-ex/0312008.

[6] S. Manly et al. [PHOBOS Collaboration], Nucl. Phys. A 774, 523 (2006) [arXiv:nucl-ex/0510031].

[7] S. A. Voloshin, A. M. Poskanzer, A. Tang and G. Wang, Phys. Lett. B 659, 537 (2008) [arXiv:0708.0800 [nucl-th]].

[8] S. Wang et al., Phys. Rev. C 44, 1091 (1991).

[9] N. Borghini, P. M. Dinh and J. Y. Ollitrault, Phys. Rev. C 64, 054901 (2001) [arXiv:nucl-th/0105040].

[10] N. Borghini, R. S. Bhalerao and J. Y. Ollitrault, J. Phys. G 30, S1213 (2004) [arXiv:nucl-th/0402053].

[11] A. M. Poskanzer and S. A. Voloshin, Phys. Rev. C 58, 1671 (1998) [arXiv:nucl-ex/9805001].

[12] P. Danielewicz and G. Odyniec, Phys. Lett. B 157 (1985) 146.

[13] J. Y. Ollitrault, arXiv:nucl-ex/9711003.

[14] J. Y. Ollitrault, Nucl. Phys. A 638, 195 (1998)

$$
\mathbf{q} \sin \Psi_{R}=\frac{\mathbf{Q}}{\sqrt{N}} \sin \Psi_{R}=\frac{1}{\sqrt{N}} \sum_{j=1}^{N} w_{j} \sin \phi_{j},(\mathrm{~B} 22)
$$

where $w_{j}$ is a weight which may depend on transverse momentum, rapidity, and mass. Using appropriate weights increases the resolution. The optimal weight is $w_{j} \propto$ $v_{2}$ [9]. A standard choice for elliptic flow at RHIC is $w=p_{T}$ up to $2 \mathrm{GeV} / c$ and flat above that.

Our discussion of fluctuations in App. A is independent of which weights are used. For nonflow effects, weights matter. The problem is that the various nonflow terms listed in this Appendix are not all weighted in the same way. More specifically, the correlation between subevents will get weights from particles from both subevents, while the correlation between the particle and the event plane only gets one weight. We note $\delta_{\text {full }}$ the nonflow correlation with one weight, and $\delta_{\text {sub }}$ the nonflow correlation with two weights. One must replace $\delta_{\text {nf }}$ with $\delta_{\text {full }}$ in Eq. (B9) and with $\delta_{\text {sub }}$ in Eqs. (B11) and (B21). Eq. (27) is then replaced by:

$$
\begin{aligned}
& v\{\mathrm{EP}\}^{2}=\langle v\rangle^{2}+\left(1-\frac{I_{0}-I_{1}}{I_{0}+I_{1}}\right) \delta_{\text {full }} \\
& -\frac{I_{0}-I_{1}}{I_{0}+I_{1}}\left(\chi^{2}-\chi_{s}^{2}+\frac{2 i_{1}^{2}}{\left(i_{0}^{2}-i_{1}^{2}\right)}\right) \delta_{\text {sub }} .
\end{aligned}
$$

[arXiv:nucl-ex/9802005].

[15] J. Y. Ollitrault, Phys. Rev. D 46, 229 (1992).

[16] C. Adler et al. [STAR Collaboration], Phys. Rev. C 66, 034904 (2002) [arXiv:nucl-ex/0206001].

[17] B. Alver et al. [PHOBOS Collaboration], Phys. Rev. Lett. 98, 242302 (2007) [arXiv:nucl-ex/0610037].

[18] R. S. Bhalerao and J. Y. Ollitrault, Phys. Lett. B 641, 260 (2006) [arXiv:nucl-th/0607009].

[19] B. Alver et al., Phys. Rev. C 77, 014906 (2008) [arXiv:0711.3724 [nucl-ex]].

[20] W. Broniowski, P. Bozek and M. Rybczynski, Phys. Rev. C 76, 054905 (2007) [arXiv:0706.4266 [nucl-th]].

[21] Hiroshi Masui, private communication, 2008.

[22] J. Adams et al. [STAR Collaboration], Phys. Rev. Lett. 93, 252301 (2004) [arXiv:nucl-ex/0407007].

[23] Aihong Tang, private communication, 2008.

[24] B. I. Abelev et al. [STAR Collaboration], Phys. Rev. C 77, 054901 (2008) [arXiv:0801.3466 [nucl-ex]].

[25] H. J. Drescher and Y. Nara, Phys. Rev. C 76, 041903 (2007) [arXiv:0707.0249 [nucl-th]].

[26] P. Filip, Phys. Atom. Nucl. 71, 1609 (2008) [arXiv:0712.0088 [nucl-th]].

[27] S. Voloshin and Y. Zhang, Z. Phys. C 70, 665 (1996) 
[arXiv:hep-ph/9407282].

[28] J. Y. Ollitrault, Phys. Rev. D 48, 1132 (1993) [arXiv:hep$\mathrm{ph} / 9303247]$.

[29] N. Borghini, P. M. Dinh, J. Y. Ollitrault,
A. M. Poskanzer and S. A. Voloshin, Phys. Rev. C 66, 014901 (2002) [arXiv:nucl-th/0202013].

[30] J. Lukasik and W. Trautmann, arXiv:nucl-ex/0603028. 